\title{
Exploring the molecular mechanisms of osteosarcoma by the integrated analysis of mRNAs and miRNA microarrays
}

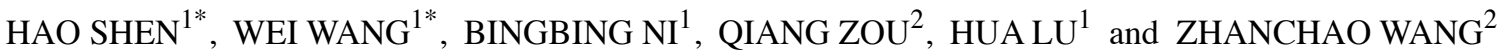 \\ ${ }^{1}$ Department of Orthopaedics, Xinhua Hospital Affiliated to Shanghai Jiaotong University School of Medicine, \\ Shanghai 200092; ${ }^{2}$ Department of Orthopaedics, Xinhua Hospital Affiliated to Shanghai Jiaotong University \\ School of Medicine, Chongming Branch, Shanghai 202150, P.R. China
}

Received December 23, 2015; Accepted August 11, 2017

DOI: $10.3892 /$ ijmm.2018.3594

\begin{abstract}
Osteosarcoma (OS) is the most frequently occurring primary bone malignancy with a rapid progression and poor survival. In the present study, in order to examine the molecular mechanisms of OS, we analyzed the microarray of GSE28425. GSE28425 was downloaded from Gene Expression Omnibus, which also included the miRNA expression profile, GSE28423, and the mRNA expression profile, GSE28424. Each of the expression profiles included 19 OS cell lines and 4 normal bones. The differentially expressed genes (DEGs) and differentially expressed miRNAs (DE-miRNAs) were screened using the limma package in Bioconductor. The DEGs associated with tumors were screened and annotated. Subsequently, the potential functions of the DEGs were analyzed by Gene Ontology (GO) and pathway enrichment analyses. Furthermore, the protein-protein interaction (PPI) network was constructed using the STRING database and Cytoscape software. Furthermore, modules of the PPI network were screened using the ClusterOne plugin in Cytoscape. Additionally, the transcription factor (TF)-DEG regulatory network, DE-miRNA-DEG regulatory network and miRNAfunction collaborative network were separately constructed to obtain key DEGs and DE-miRNAs. In total, 1,609 DEGs
\end{abstract}

Correspondence to: Dr Hua Lu, Department of Orthopaedics, Xinhua Hospital Affiliated to Shanghai Jiaotong University School of Medicine, 1665 Kongjiang Road, Shanghai 200092, P.R. China E-mail:1hhawk@hotmail.com

Dr Zhanchao Wang Department of Orthopaedics, Xinhua Hospital Affiliated to Shanghai Jiaotong University School of Medicine, Chongming Branch. 25 Nanmeng Road, Chengqiao Town, Chongming County, Shanghai 202150, P.R. China

E-mail: 1cwzc@126.com

${ }^{*}$ Co-first authorship

Key words: osteosarcoma, differentially expressed genes, differentially expressed miRNAs, protein-protein interaction network, regulatory network, miRNA-function collaborative network and 149 DE-miRNAs were screened. Upregulated FOS-like antigen 1 (FOSL1) also had the function of an oncogene. MAD2 mitotic arrest deficient-like 1 (MAD2L1; degree, 65) and aurora kinase A (AURKA; degree, 64) had higher degrees in the PPI network of the DEGs. In the TF-DEG regulatory network, the TF, signal transducer and activator of transcription 3 (STAT3) targeted the most DEGs. Moreover, in the DE-miRNA-DEG regulatory network, downregulated $m i R-1$ targeted many DEGs and estrogen receptor 1 (ESRI) was targeted by several highly expressed miRNAs. Moreover, in the miRNA-function collaborative networks of upregulated miRNAs, $m i R-128$ targeted myeloid dendritic associated functions. On the whole, our data indicate that MAD2L1, AURKA, STAT3, ESR1, FOSL1, miR-1 and miR-128 may play a role in the development and/or progressio of OS.

\section{Introduction}

As a high-grade type and mesenchymally-derived bone sarcoma (1), osteosarcoma (OS) is the most prevalent primary bone cancer and the 8th most frequent type of cancer affecting young patients (2). Being characterized by a high malignant degree, rapid progression and a poor survival, OS consists up to $15 \%$ of all solid extracranial cancers in patients aged $15-19$ years $(3,4)$. Thus, it is necessary to identify biomarkers involved in OS.

DNA repair gene RecQ protein-like 4 (RECQL4) is overexpressed in OS, and its overexpression is related to overall genomic instability (5). Human epidermal growth factor receptor 2 (Her-2/neu) expression can induce lung metastasis in OS and may be related to gene amplification (6). Overexpressed c-fos $(F O S)$ and runt-related transcription factor 2 (RUNX2) may play a role in OS; in particular, RUNX2 expression may serve as a marker of chemotherapy failure in patients with OS $(7,8)$. The cell cycle regulator, CDC5 cell division cycle 5-like $(C D C 5 L)$, is essential for the $\mathrm{G} 2-\mathrm{M}$ transition and may be potential oncogene for the 6p12-p21 amplicon in OS (9). It has been reported that genes with the function of transcription factors (TFs) can also play a role in OS, such as Yin Yang $1(Y Y 1)$, which is expressed in the early process of osteoblastic transformation and its detection may be used as a promising diagnostic method in human OS (10). In addition, the TF osterix $(O s x)$ can suppress the lung migration of OS 
tumor cells; thus, the expression of Osx may be implicated in the growth and metastasis of OS (11).

There are also many studies which have investigated the direct or indirect effect of microRNAs (miRNAs or miRs) on OS. For example, by targeting matrix metalloprotease 13 (MMP13) and B-cell CLL/lymphoma 2 (Bcl-2), miR-143 may be involved in the lung metastasis of human OS cells and may thus be used as a target in cancer therapy $(12,13)$. In addition, downregulated $m i R-199 a-3 p$ may function in the growth and proliferation of OS cells; hence, restoring the function of $m i R-199 a-3 p$ may contribute to the treatment of OS (14). By mediating reversioninducing-cysteine-rich protein with kazal motifs (RECK), $m i R-21$ plays an important role in regulating cell invasion and migration in OS and may be a potential therapeutic target (15). By regulating $c$-Met and other genes, $m i R-34 a$ can function as a tumor suppressor gene and suppresses the pulmonary metastasis of OS; thus, it may be a useful gene therapeutic agent (16).

In 2012, Namløs et al (17) used global microarray analyses to identify the differentially expressed miRNAs (DE-miRNAs) between OS cell lines and normal bones, and obtained 177 DE-miRNAs. In this study, using the same data by Namløs et al (17), we aimed to further screen the differentially expressed genes (DEGs) and DE-miRNAs. The potential functions of the DEGs were analyzed by Gene Ontology (GO) and Kyoto Encyclopedia of Genes and Genomes (KEGG) pathway enrichment analyses. Subsequently, the interaction associations of the proteins encoded by the DEGs were investigated by protein-protein interaction network (PPI) network and modules of PPI network. In addition, the TF-DEG regulatory network, DE-miRNA-DEG regulatory network and miRNA-function collaborative network were separately constructed to obtain key DEGs and DE-miRNAs.

\section{Data collection methods and analysis}

Microarray data. The microarray of GSE28425 deposited by Namløs et al (17) was downloaded from Gene Expression Omnibus (GEO, http://www.ncbi.nlm.nih.gov/geo/), which included the miRNA expression profile, GSE28423, and the mRNA expression profile, GSE28424. Each of the expression profiles included a collection of 19 OS cell lines and 4 normal bones. GSE28423 was based on the platform of GPL8227 Agilent-019118 Human miRNA Microarray 2.0 G4470B (Agilent Technologies Inc., Santa Clara, CA, USA). GSE28424 was based on the platform of GPL13376 Illumina HumanWG-6 v2.0 expression beadchip (Illumina, San Diego, CA, USA). The OS samples were from a panel collected within EuroBoNeT and from the Norwegian Radium Hospital. Meanwhile, normal bones were from Capital Biosciences or from amputations of cancer patients at the University College London and Norwegian Radium Hospital.

Screening of DEGs and DE-miRNAs. After GSE28425 was downloaded, the microarray data was pre-processed using the limma package (18) in Bioconductor (http://www.bioconductor. org/packages/release/bioc/html/limma.html). In brief, the preprocessing process included Background Correction, Quantile Normalization and Probe Summarization. The limma (linear models for microarray data) package (18) was used to analyze the DEGs and DE-miRNAs between the OS cell lines and normal bones. The FDR (that is, adjusted p-value) $<0.05$ and $\mid \log 2$ fold-change (FC) $\mid>1$ were used as the cut-off criteria. Screening the tumor suppressor (TS) gene (http://bioinfo. mc.vanderbilt.edu/TSGene/download.cgi) (19) and tumorassociated gene (TAG) (http://www.binfo.ncku.edu.tw/TAG/ GeneDoc.php) (20) databases, the DEGs associated with tumors were screened and annotated.

Functional and pathway enrichment analysis. GO provides controlled and structured vocabularies which model biological process (BP), cell components (CC) and molecular function (MF) (21). KEGG is a database containing 16 main databases, roughly divided into systems information, chemical information and genomic information (22). GO functional enrichment analyses, which involved the $\mathrm{BP}, \mathrm{MF}$ and $\mathrm{CC}$ categories, as well as KEGG pathway enrichment analyses were performed for the DEGs and the DE-miRNAs. A p-value $<0.05$ was used as the cut-off criterion.

PPI network and module construction. The interaction associations of the proteins encoded by the DEGs were searched using STRING online software (http://string-db.org; v9.05) (23), and the combined score of $>0.7$ was used as the cut-off criterion. The PPI network was visualized using Cytoscape software (http:// www.cytoscape.org) (24). Modules of the PPI network were screened using the ClusterOne plugin (25) in Cytoscape, and the significant p-value of the modules were set to $1.1 \mathrm{E}-6$.

TF-DEG regulatory network construction. Human TF-gene regulatory pairs were downloaded from the UCSC database (http://genome.ucsc.edu/) (26). The DEGs which can also function as TFs and their target genes were then identified. Moreover, Cytoscape software (24) was used to visualize the the TF-DEG regulatory network.

DE-miRNA-DEG regulatory network construction. By comparing the experimental validated miRNA-mRNA pairs in the miRecords (http://www.mirecords.umn.edu) (27) and mirWalk (http://mirwalk.uni-hd.de/) (28) databases, pairs of DE-miRNAs from the miRNA expression profile, GSE28423, and DEGs from the mRNA expression profile, GSE28424, were obtained. The DE-miRNA-DEG pairs should appear in either miRecords database or mirWalk database.

miRNA-function collaborative network construction. According to the functional enrichment results of the DE-miRNAs, the DE-miRNAs which targeted the genes involved in one BP term were identified. Subsequently, miRNA-function collaborative network was constructed. A p-value $<0.01$ was used as the cut-off criterion.

\section{Results}

DEGs analysis. Compared with normal bones, there were 1,609 DEGs (including 774 upregulated and 835 downregulated mRNAs) and 149 DE-miRNAs (including 76 upregulated and 73 downregulated miRNAs) screened in the OS cell lines. The DEGs associated with tumors were annotated and are listed in Table I. Importantly, upregulated FOS-like antigen 1 (FOSL1) also had the function of an oncogene. 
Table I. The DEGs associated with tumors.

\begin{tabular}{|c|c|c|c|}
\hline Category & Oncogene & $\mathrm{TSG}$ & TAG \\
\hline UP & $\begin{array}{l}\text { CDC5L, FOSL1, HMMR, } \\
\text { AURKA, MLF1, CDK4, } \\
\text { MET, TRIO, NRAS, } \\
\text { HOXA10, WHSC1, } \\
\text { PIK3CA }\end{array}$ & $\begin{array}{l}\text { S100A2, TUSC3, PAWR, LZTS1, YAP1, } \\
\text { GADD45GIP1, PTPRG, RND3, DFNA5, } \\
\text { HOXB13, BAI2, ZDHHC2, NF2, BCL10, } \\
\text { FANCG, AMH, RCN2, HLTF, NME1, REV3L, } \\
\text { DAPK3, FH, MEN1, HECA, TRIM3, } \\
\text { SCRIB, BRMS1, EXTL3, SMARCB1, PCGF2 }\end{array}$ & $\begin{array}{l}\text { TFAP } 2 A, B U B 1, N K X 3-1, \\
D N M T 3 B, P M S 1, \\
\text { SHC1, YEATS4, } \\
\text { FADD, C1QBP }\end{array}$ \\
\hline DOWN & $\begin{array}{l}F G F 20, L Y N, B C L 6, \\
T A L 1, E S R 1, \text { WISP2, } \\
\text { LMO2, LCN2, LYL1 }\end{array}$ & $\begin{array}{l}\text { HSD17B7, PRODH, MAL, DUSP22, TSC22D1, } \\
\text { COL4A3, BAI3, BNIP3L, PER1, PAEP, RASSF4, } \\
\text { FOXC1, EXTL1, ARHGAP20, CMTM5, NGFR, } \\
\text { TXNIP, NOTCH1, MRVI1, MTSS1, MTUS1, PPAP2A, } \\
\text { TCF4, ST5, PYHIN1, PRKCD, TGFBR3, CBFA2T3, } \\
\text { MT1G, TSPAN32, RASSF2, CEBPA, LTF, RARRES1, } \\
\text { MAP4K1, BTG2, PLA2G2A, ZBTB16, SYK, } \\
\text { GPX3, PYCARD, H19, PTPN6, C2orf40 }\end{array}$ & $\begin{array}{l}\text { TAL2, WISP3, STAT3, } \\
C B L B, N R 4 A 2, L Y S T, \\
R G S 2, F E S, M G P\end{array}$ \\
\hline
\end{tabular}

TSG, tumor suppressor gene; TAG, tumor-associated gene.

Functional and pathway enrichment analysis. The top 5 enriched GO functions in the BP, CC and MF categories separately for the upregulated and downregulated genes are listed in Table II. For the upregulated genes, the enriched functions included cell cycle $(\mathrm{p}=0)$, intracellular membrane-bounded organelle $(p=0)$ and catalytic activity $(p=3.05 E-10)$. For the downregulated genes, the enriched functions included cell activation $(\mathrm{p}=0)$, extracellular region $(\mathrm{p}=0)$ and carbohydrate derivative binding $(\mathrm{p}=1.55 \mathrm{E}-08)$.

The top 10 enriched KEGG pathways separately for the upregulated and downregulated genes are also listed in Table II. For the upregulated genes, the enriched pathways included metabolic pathways $(\mathrm{p}=1.14 \mathrm{E}-06)$, steroid biosynthesis $(p=2.02 E-05)$ and spliceosome $(p=0.003765459)$. For the downregulated genes, the enriched pathways included cytokine-cytokine receptor interaction $(\mathrm{p}=3.76 \mathrm{E}-06)$ and osteoclast differentiation $(\mathrm{p}=7.87 \mathrm{E}-06)$.

PPInetwork and module analysis. The PPI network of the DEGs had 844 nodes and 3,400 interactions. In particular, MAD2 mitotic arrest deficient-like 1 (MAD2L1, degree, 65), cyclin B1 (CCNB1, degree, 65) and aurora kinase A (AURKA, degree, 64) had high degrees in the PPI network. In addition, 3 modules (module 1, module 2 and module 3 ) of the PPI network were screened (Fig. 1). In module 1, TAO kinase 1 (TAOK1) was the only downregulated gene. The enriched KEGG pathways for the DEGs in module 1 included oocyte meiosis $(\mathrm{p}=2.04 \mathrm{E}-08)$, cell cycle $(\mathrm{p}=4.16 \mathrm{E}-08)$ and progesterone-mediated oocyte maturation ( $\mathrm{p}=0.000112373$ ) (Table III). In module 2, guanine nucleotide binding protein, $\alpha$ inhibiting 1 (GNAI1) and regulator of G-protein signaling 20 (RGS20) were downregulated. The enriched KEGG pathways for the DEGs in module 2 included chemokine signaling pathway $(\mathrm{p}=0)$ and cytokine-cytokine receptor interaction $(\mathrm{p}=9.77 \mathrm{E}-15)$ (Table III). Furthermore, the
DEGs involved in module 3 were all upregulated genes. The enriched KEGG pathways for the DEGs in module 3 included ribosome $(\mathrm{p}=1.26 \mathrm{E}-12)$ and protein processing in endoplasmic reticulum $(\mathrm{p}=0.043084724)$ (Table III).

TF-DEG regulatory network analysis. The TF-DEG regulatory network had 311 interactions (involving 10 transcription factors and 285 DEGs) (Fig. 2). Importantly, the TFs, signal transducer and activator of transcription 3 (STAT3, degree, 158) and forkhead box A1 (FOXA1, degree, 106) targeted the most DEGs.

DE-miRNA-DEG regulatory network analysis. The DE-miRNA-DEG regulatory network involved 23 upregulated miRNAs and 64 downregulated miRNAs (Fig. 3). In the DE-miRNA-DEG regulatory network, downregulated $m i R-1$ targeted and activated many DEGs. Moreover, downregulated estrogen receptor 1 (ESRI) was targeted by several high-expressed miRNAs, including miR-221, miR-20b and miR-18a. The enriched GO functions for the upregulated and downregulated miRNAs are listed in Table IV. For the upregulated miRNAs, the enriched functions included positive regulation of retinoic acid receptor signaling pathway $(\mathrm{p}=0.000211583)$ and type 1 metabotropic glutamate receptor binding $(\mathrm{p}=0.000150457)$. For the downregulated miRNAs, the enriched functions included response to inactivity $(\mathrm{p}=0.001989302)$ and potassium ion binding $(\mathrm{p}=0.006643278)$.

miRNA-function collaborative network analysis. The miRNA-function collaborative networks of upregulated (Fig. 4) and downregulated (Fig. 5) miRNAs were constructed, respectively. In the miRNA-function collaborative networks of upregulated miRNAs, myeloid dendritic associated functions were targeted by miR-128 and miR-125a-5p. 


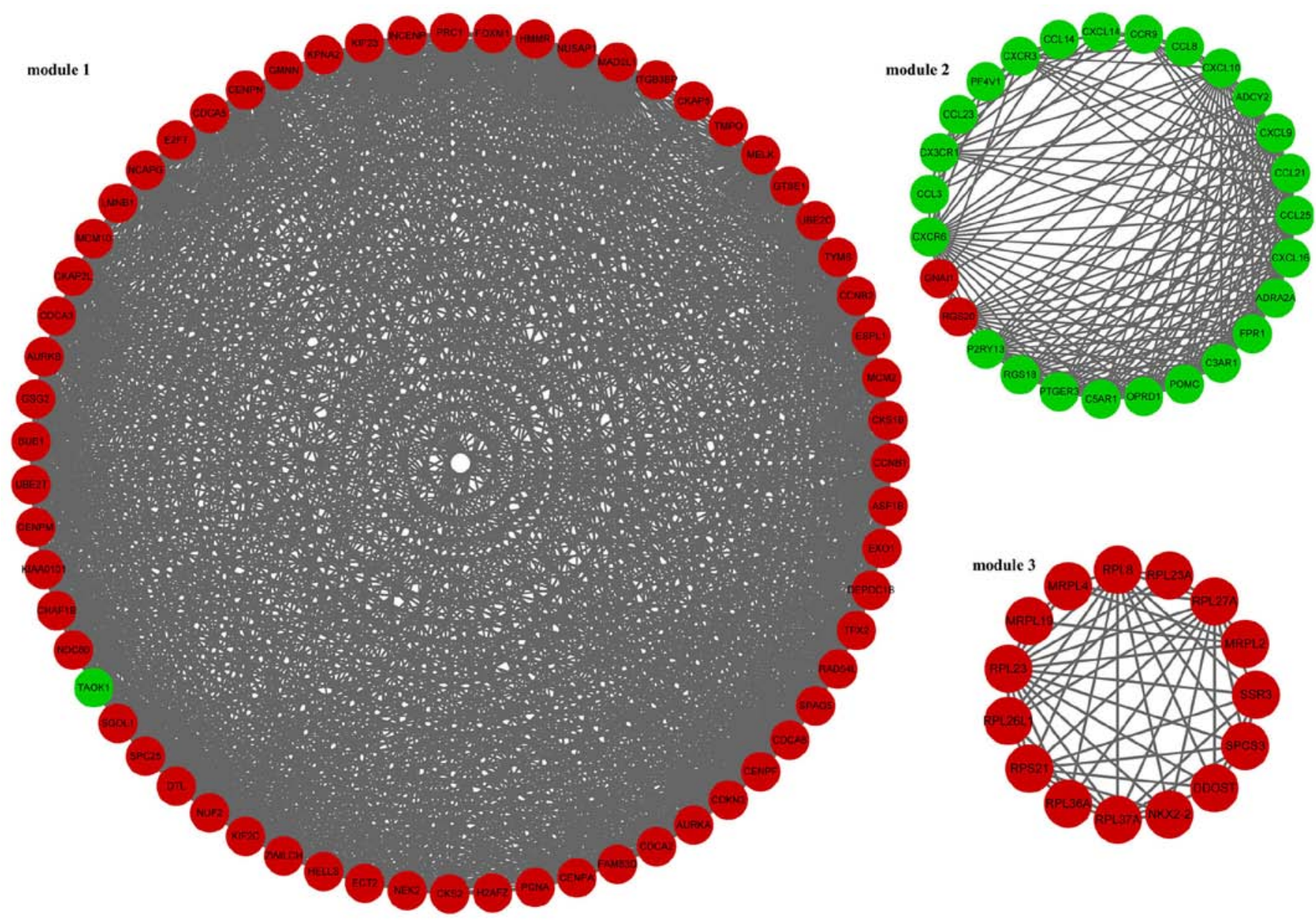

Figure 1. The three modules (module 1, module 2 and module 3) screened from the protein-protein interaction (PPI) network. The red circle nodes represent the upregulated genes, while the green circle nodes represent the downregulated genes.

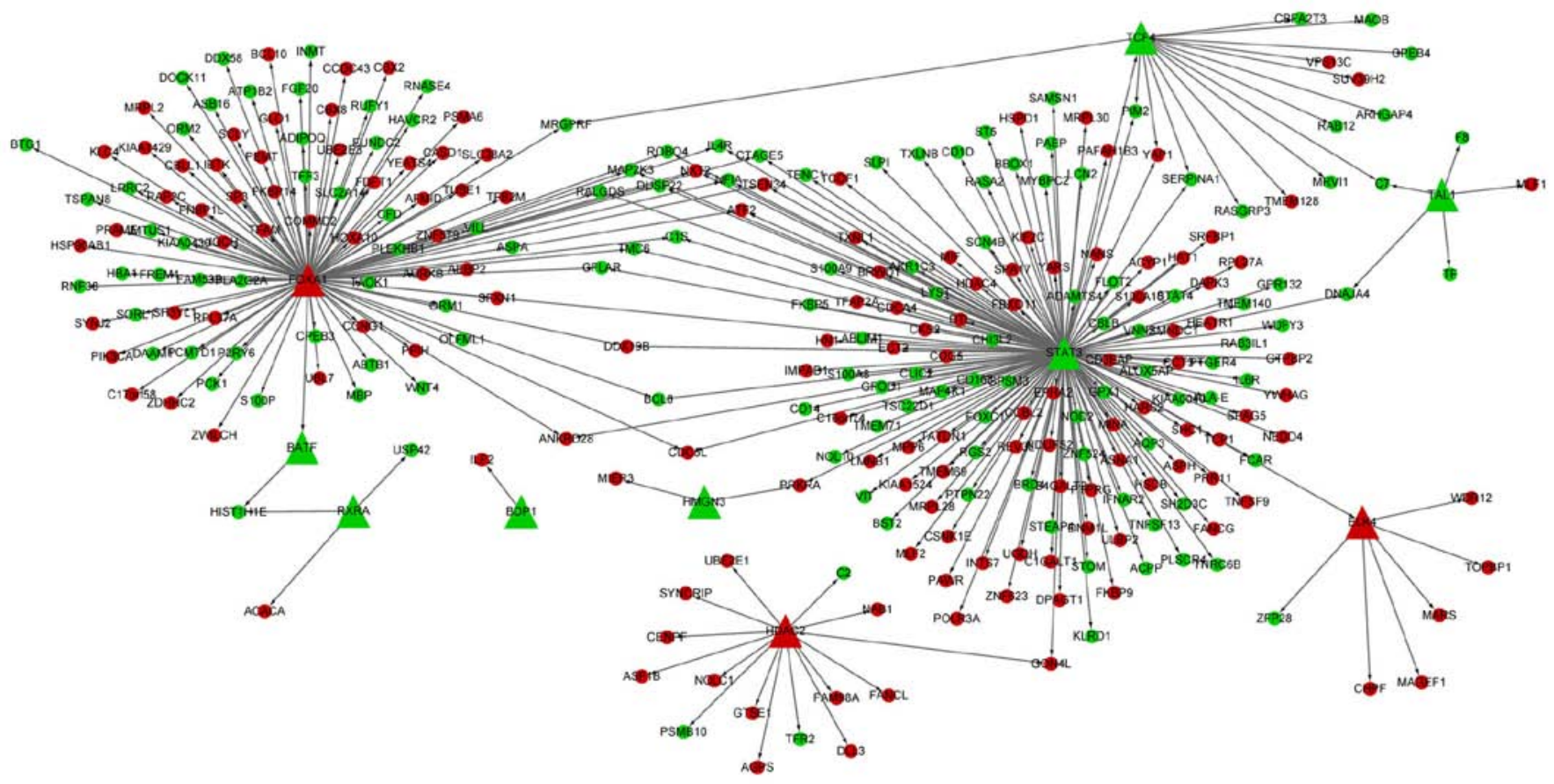

Figure 2. The TF-differentially expressed genes (DEG) regulatory network. The red nodes represent the upregulated genes, while the green nodes represent the downregulated genes. In addition, the triangle nodes stand for transcription factors, and circles nodes stand for their targeted genes. 
Table II. The top 5 enriched GO functions in BP, CC and MF categories, as well as the top 10 enriched KEGG pathways separately for the upregulated and downregulated genes.

\begin{tabular}{|c|c|c|c|c|c|}
\hline Category & Term & Description & Gene no. & Gene symbol & p-value \\
\hline \multirow[t]{5}{*}{ UP_BP } & GO:0007049 & Cell cycle & 133 & KPNA2, UBE2C & 0 \\
\hline & GO:0000278 & Mitotic cell cycle & 90 & $C D C A 3, E 2 F 7$ & $2.22 \mathrm{E}-16$ \\
\hline & GO:0022402 & Cell cycle process & 108 & $F A M 83 D, S P C 25$ & $1.22 \mathrm{E}-15$ \\
\hline & GO:0051301 & Cell division & 62 & $U B E 2 C, C D C A 3$ & $3.86 \mathrm{E}-14$ \\
\hline & GO:0048285 & Organelle fission & 52 & $F A M 83 D, S P C 25$ & $7.72 \mathrm{E}-14$ \\
\hline \multirow[t]{5}{*}{$\mathrm{UP} \_\mathrm{CC}$} & GO:0005622 & Intracellular & 611 & $T F A P 2 A, C B S$ & 0 \\
\hline & GO:0031981 & Nuclear lumen & 161 & $C B S, K P N A 2$ & 0 \\
\hline & GO:0043231 & $\begin{array}{l}\text { Intracellular membrane- } \\
\text { bounded organelle }\end{array}$ & 509 & KPNA2, JPH3 & 0 \\
\hline & GO:0044422 & Organelle part & 384 & SHROOM3, UBE2C & 0 \\
\hline & GO:0044424 & Intracellular part & 607 & $F O X D 1, U B E 2 C$ & 0 \\
\hline \multirow[t]{5}{*}{ UP_MF } & GO:0003824 & Catalytic activity & 300 & PSAT1, UBE2C & $3.05 \mathrm{E}-10$ \\
\hline & GO:0016740 & Transferase activity & 123 & PSAT1, CCNB1 & 3.95E-09 \\
\hline & GO:0005515 & Protein binding & 382 & $T F A P 2 A, C B S$ & $1.13 \mathrm{E}-07$ \\
\hline & GO:0032549 & Ribonucleoside binding & 115 & $U B E 2 C, K I F 2 C$ & $3.57 \mathrm{E}-06$ \\
\hline & GO:0035639 & $\begin{array}{l}\text { Purine ribonucleoside } \\
\text { triphosphate binding }\end{array}$ & 114 & SEPT3, PTK7 & 4.27E-06 \\
\hline \multirow[t]{5}{*}{ DOWN_BP } & GO:0001775 & Cell activation & 104 & $G R A P 2, I L 12 R B 1$ & 0 \\
\hline & GO:0001816 & Cytokine production & 73 & STAT5B, LIPA & 0 \\
\hline & GO:0002376 & Immune system process & 252 & $F G F 20, F C G R 3 A$ & 0 \\
\hline & GO:0002682 & Regulation of immune system process & 153 & $B L K, C D 200 R 1$ & 0 \\
\hline & GO:0002684 & $\begin{array}{l}\text { Positive regulation of immune } \\
\text { system process }\end{array}$ & 99 & $F C G R 3 A, G R A P 2$ & 0 \\
\hline \multirow[t]{5}{*}{ DOWN_CC } & GO:0005576 & Extracellular region & 191 & $F G F 20, F C G R 3 A$ & 0 \\
\hline & GO:0005615 & Extracellular space & 98 & CCL25, APOC2 & 0 \\
\hline & GO:0005886 & Plasma membrane & 316 & $I L 12 R B 1, B L K$ & 0 \\
\hline & GO:0044421 & Extracellular region part & 120 & $I L 12 R B 1, B L K$ & 0 \\
\hline & GO:0044459 & Plasma membrane part & 174 & OPRD1, MAL & 0 \\
\hline \multirow[t]{5}{*}{ DOWN_MF } & GO:0097367 & Carbohydrate derivative binding & 29 & $F G F 7, T L R 2$ & $1.55 \mathrm{E}-08$ \\
\hline & GO:0005515 & Protein binding & 417 & $F G F 20, H M G N 3$ & 3.54E-08 \\
\hline & GO:0046983 & Protein dimerization activity & 80 & $A D D 2, A P O C 2$ & $5.30 \mathrm{E}-08$ \\
\hline & GO:0008307 & Structural constituent of muscle & 13 & $D M D, M Y L 4$ & $9.25 \mathrm{E}-08$ \\
\hline & GO:0042803 & Protein homodimerization activity & 55 & $M Z F 1, A D D 1$ & $1.31 \mathrm{E}-07$ \\
\hline $\mathrm{UP}_{-}$ & 01100 & Metabolic pathways & 89 & CBS, PSAT1 & $1.14 \mathrm{E}-06$ \\
\hline \multirow[t]{9}{*}{ KEGG } & 00100 & Steroid biosynthesis & 7 & DHCR24, SQLE & 2.02E-05 \\
\hline & 03040 & Spliceosome & 14 & $C D C 5 L, S M N D C 1$ & 0.003765459 \\
\hline & 03008 & Ribosome biogenesis in eukaryotes & 10 & $N X T 2, N M D 3$ & 0.005664746 \\
\hline & 00270 & Cysteine and methionine metabolism & 6 & $C B S, D N M T 3 B$ & 0.007673661 \\
\hline & 00510 & N-Glycan biosynthesis & 7 & TUSC3, ALG10B & 0.009627025 \\
\hline & 00970 & Aminoacyl-tRNA biosynthesis & 8 & $M A R S, Y A R S$ & 0.011699718 \\
\hline & 00620 & Pyruvate metabolism & 6 & $M E 1, A C A T 2$ & 0.012824724 \\
\hline & 00290 & Valine, leucine and isoleucine biosynthesis & 3 & BCAT1, VARS, LARS & 0.014647016 \\
\hline & 01040 & Biosynthesis of unsaturated fatty acids & 4 & $\begin{array}{l}\text { PTPLA, ELOVL5, } \\
P T P L B, S C D\end{array}$ & 0.017934809 \\
\hline DOWN_ & 05150 & Staphylococcus aureus infection & 21 & $F C A R, C 3 A R 1$ & 2.29E-12 \\
\hline \multirow[t]{4}{*}{ KEGG } & 04640 & Hematopoietic cell lineage & 21 & $I L A R, C R I$ & 4.15E-08 \\
\hline & 04145 & Phagosome & 27 & $T L R 2, N O X 1$ & 4.14E-07 \\
\hline & 05140 & Leishmaniasis & 17 & CRI,IFNGRI & $9.79 \mathrm{E}-07$ \\
\hline & 04060 & Cytokine-cytokine receptor interaction & 36 & CCL25, TNFSF8 & $3.76 \mathrm{E}-06$ \\
\hline
\end{tabular}


Table II. Continued.

\begin{tabular}{lllrlr}
\hline Category & Term & \multicolumn{1}{c}{ Description } & Gene no. & Gene symbol & p-value \\
\hline 04380 & Osteoclast differentiation & 22 & LILRA6, NOX1 & $7.87 \mathrm{E}-06$ \\
04650 & Natural killer cell mediated cytotoxicity & 22 & IFNGR1, NFATC3 & $2.14 \mathrm{E}-05$ \\
04514 & Cell adhesion molecules (CAMs) & 21 & MAG, F11R & $4.77 \mathrm{E}-05$ \\
05310 & Asthma & 9 & $M S 4 A 2, E P X$ & $4.91 \mathrm{E}-05$ \\
05416 & Viral myocarditis & 14 & DMD, SGCA & $6.63 \mathrm{E}-05$ \\
\hline
\end{tabular}

GO, Gene Ontology; BP, biological process; CC, cell components; MF, molecular function; KEGG, Kyoto Encyclopedia of Genes and Genomes .

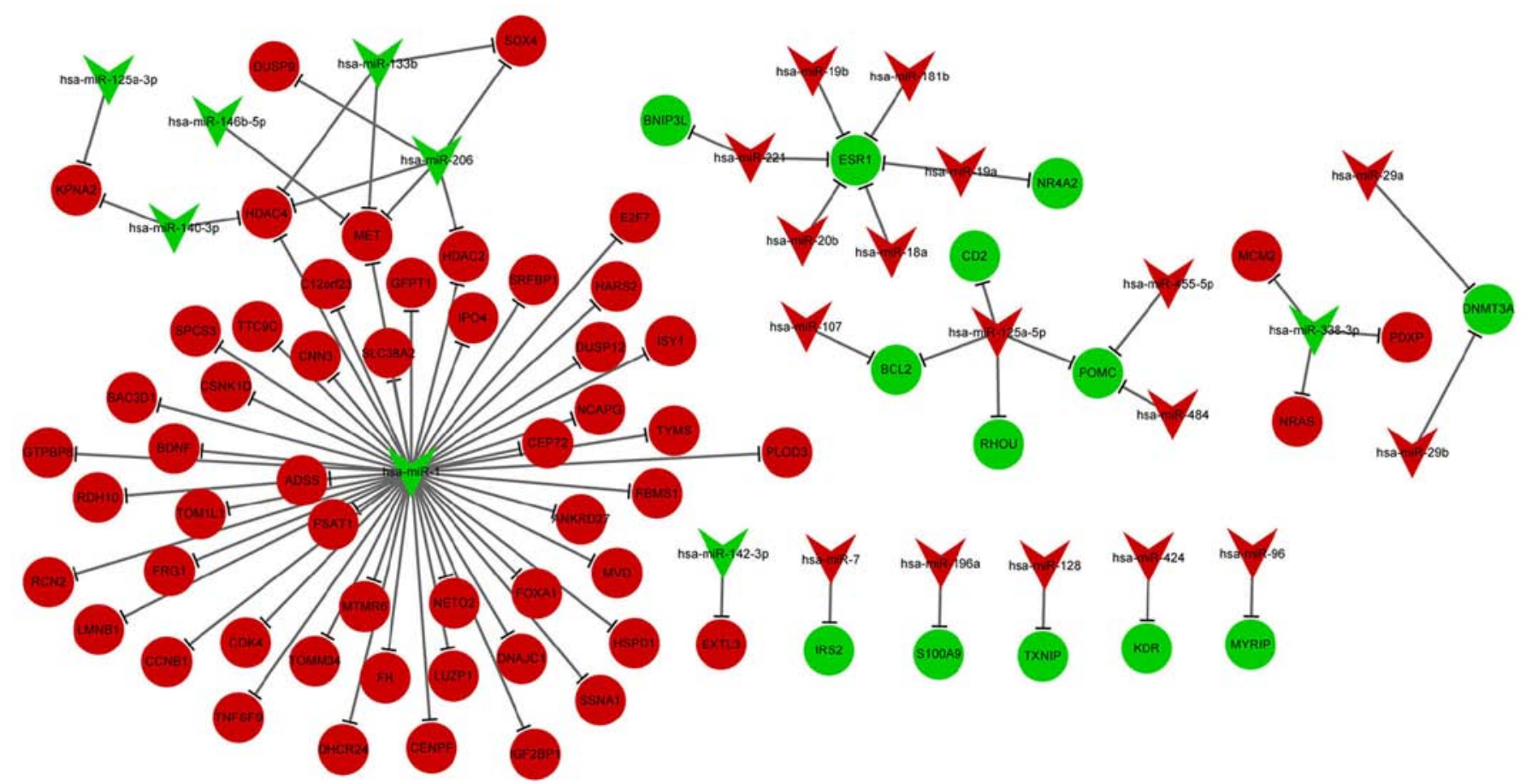

Figure 3. The DE-miRNA-DEG regulatory network. The red nodes represent the upregulated DEGs, while the green nodes represent the downregulated DEGs. In addition, the nodes in inverse triangle stand for miRNAs, and circles nodes stand for their targeted genes. DEGs: differentially expressed genes.

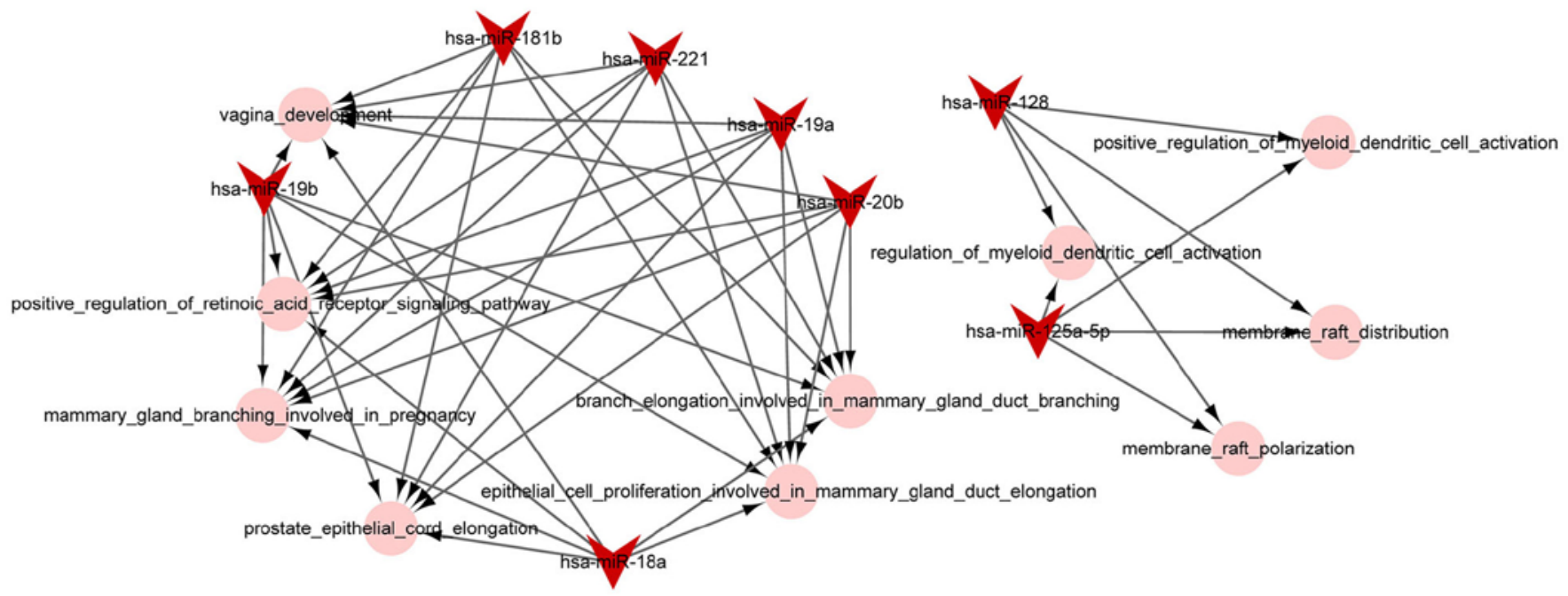

Figure 4. The miRNA-function collaborative network of upregulated miRNAs. The pink circle nodes represent the biological process terms of Gene Ontology. 
Table III. The enriched KEGG pathways for the DEGs in module 1, module 2 and module 3 of the PPI network.

\begin{tabular}{|c|c|c|c|c|c|}
\hline & Term & Description & Gene no. & Gene symbol & p-value \\
\hline \multirow[t]{6}{*}{ Module 1} & 04114 & Oocyte meiosis & 7 & $A U R K A, S G O L 1$ & 2.04E-08 \\
\hline & 04110 & Cell cycle & 7 & $P C N A, M C M 2$ & 4.16E-08 \\
\hline & 04914 & Progesterone-mediated oocyte maturation & 4 & $\begin{array}{l}C C N B 2, B U B 1, \\
M A D 2 L 1, C C N B 1\end{array}$ & 0.000112373 \\
\hline & 04115 & p53 signaling pathway & 3 & $C C N B 2, C C N B 1, G T S E 1$ & 0.001071355 \\
\hline & 03430 & Mismatch repair & 2 & PCNA, EXO1 & 0.002163033 \\
\hline & 03030 & DNA replication & 2 & PCNA, MCM2 & 0.00526065 \\
\hline \multirow[t]{9}{*}{ Module 2} & 04062 & Chemokine signaling pathway & 17 & $A D C Y 2, C X 3 C R 1$ & 0 \\
\hline & 04060 & Cytokine-cytokine receptor interaction & 15 & CX3CR1, CXCR6 & 9.77E-15 \\
\hline & 04080 & Neuroactive ligand-receptor interaction & 7 & OPRD1, P2RY13 & $9.93 \mathrm{E}-05$ \\
\hline & 05150 & Staphylococcus aureus infection & 3 & C3AR1, FPR1,C5AR1 & 0.001547235 \\
\hline & 04916 & Melanogenesis & 3 & ADCY2, POMC, GNAII & 0.008639105 \\
\hline & 04620 & Toll-like receptor signaling pathway & 3 & CCL3, CXCL9, CXCL1O & 0.008876016 \\
\hline & 04672 & Intestinal immune network for IgA production & 2 & CCL25, CCR9 & 0.017427247 \\
\hline & 04610 & Complement and coagulation cascades & 2 & $C 3 A R 1, C 5 A R 1$ & 0.034329309 \\
\hline & 04971 & Gastric acid secretion & 2 & $A D C Y 2, G N A I 1$ & 0.039017822 \\
\hline \multirow[t]{2}{*}{ Module 3} & 03010 & Ribosome & 8 & RPL27A, RPL37A & $1.26 \mathrm{E}-12$ \\
\hline & 04141 & Protein processing in endoplasmic reticulum & 2 & DDOST, SSR3 & 0.043084724 \\
\hline
\end{tabular}

KEGG, Kyoto Encyclopedia of Genes and Genomes; DEGs, differentially expressed genes; PPI, protein-protein interaction.

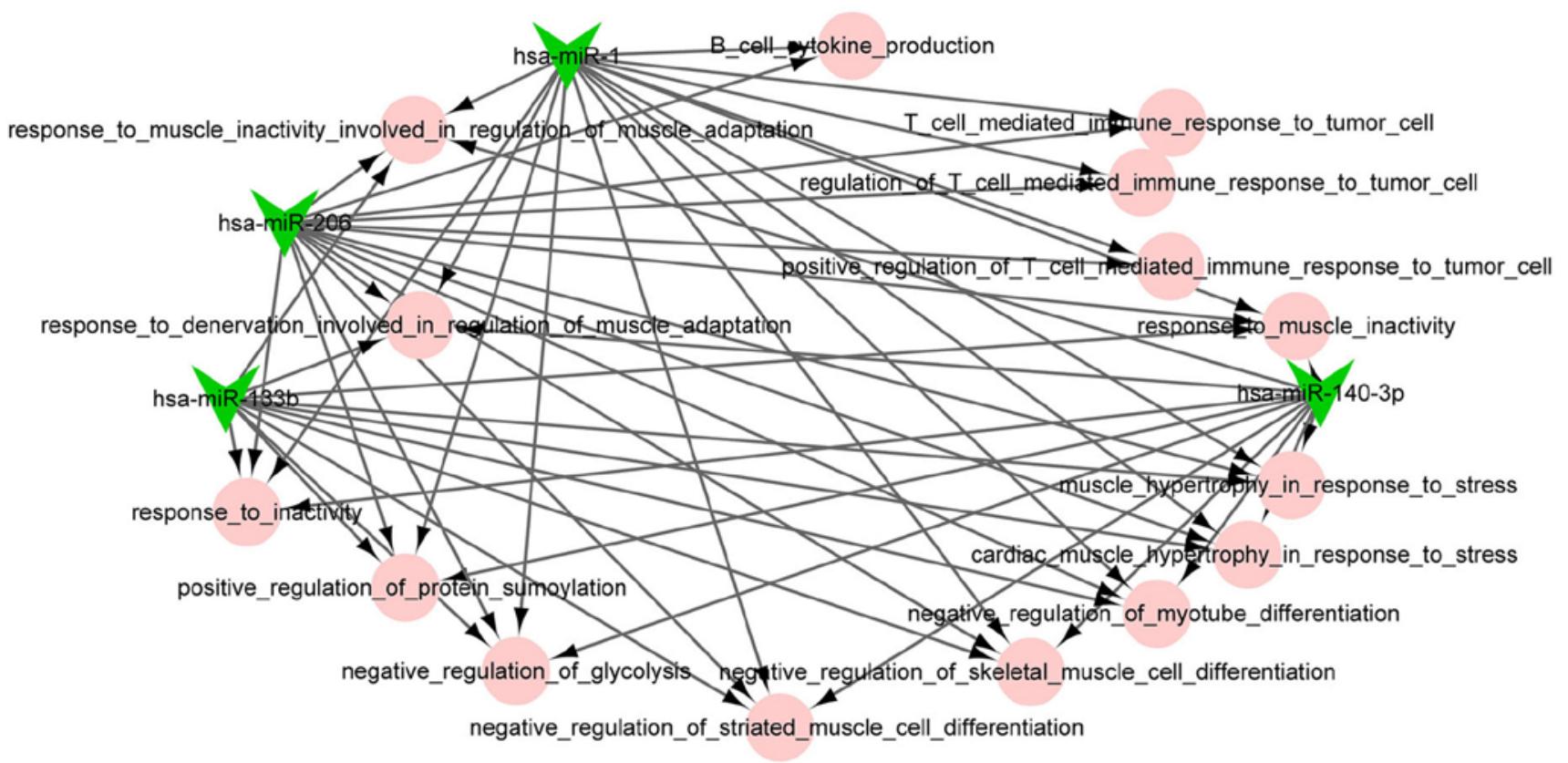

Figure 5. The miRNA-function collaborative network of downregulated miRNAs. The pink circle nodes represent the biological process terms of Gene Ontology.

\section{Discussion}

In this study, we screened 1,609 DEGs (including 774 upregulated and 835 downregulated mRNAs) and 149 DE-miRNAs (including 76 upregulated and 73 downregulated miRNAs) in the OS cell lines compared with normal bones. Importantly, upregulated FOSL1 also had the function of an oncogene.
MAD2L1 (degree, 65) and AURKA (degree, 64) had higher degrees in the PPInetwork of the DEGs.In the DE-miRNA-DEG regulatory network, downregulated $m i R-1$ targeted many DEGs and ESRl were targeted by several highly expressed miRNAs. Moreover, in the miRNA-function collaborative networks of upregulated miRNAs, $m i R-128$ targeted myeloid dendritic associated functions. 
Table IV. The enriched GO functions for the upregulated and downregulated miRNAs involved in the DE-miRNA-DEG regulatory network.

\begin{tabular}{|c|c|c|c|c|c|}
\hline Category & Term & Description & $\begin{array}{l}\text { miRNA } \\
\text { no. }\end{array}$ & $\begin{array}{l}\text { miRNA } \\
\text { symbol }\end{array}$ & p-value \\
\hline \multirow[t]{6}{*}{ UP_BP } & 0048386 & $\begin{array}{l}\text { Positive regulation of retinoic acid } \\
\text { Receptor signaling pathway }\end{array}$ & 6 & $m i R-221, m i R-18 a$ & 0.000211583 \\
\hline & 0060523 & Prostate epithelial cord elongation & 6 & $m i R-20 b, m i R-18 a$ & 0.001058491 \\
\hline & 0060745 & $\begin{array}{l}\text { Mammary gland branching involved } \\
\text { in pregnancy }\end{array}$ & 6 & $m i R-221, m i R-20 b$ & 0.001196154 \\
\hline & 0001766 & Membrane raft polarization & 2 & $m i R-125 a-5 p, m i R-128$ & 0.002429205 \\
\hline & 0030885 & $\begin{array}{l}\text { Regulation of myeloid dendritic } \\
\text { cell activation }\end{array}$ & 2 & $m i R-125 a-5 p, m i R-128$ & 0.002429205 \\
\hline & 0030887 & $\begin{array}{l}\text { Positive regulation of myeloid dendritic } \\
\text { cell activation }\end{array}$ & 2 & $m i R-125 a-5 p, m i R-128$ & 0.002429205 \\
\hline \multirow[t]{7}{*}{ UP_MF } & 0031798 & $\begin{array}{l}\text { Type } 1 \text { metabotropic glutamate } \\
\text { receptor binding }\end{array}$ & 6 & $m i R-221, m i R-20 b$ & 0.000150457 \\
\hline & 0030235 & Nitric-oxide synthase regulator activity & 6 & $m i R-19 b, m i R-20 b$ & 0.000413057 \\
\hline & 0035256 & $\begin{array}{l}\text { G-protein coupled glutamate } \\
\text { receptor binding }\end{array}$ & 6 & $m i R-19 b, m i R-18 a$ & 0.000413057 \\
\hline & 0030284 & Estrogen receptor activity & 6 & $m i R-19 a, m i R-18 a$ & 0.002145221 \\
\hline & 0034056 & Estrogen response element binding & 6 & $m i R-19 b, m i R-19 a$ & 0.003216968 \\
\hline & 0031779 & Melanocortin receptor binding & 3 & $\begin{array}{l}m i R-455-5 p \\
m i R-125 a-5 p \\
m i R-484\end{array}$ & 0.009585627 \\
\hline & 0031781 & Type 3 melanocortin receptor binding & 3 & $\begin{array}{l}\operatorname{miR}-455-5 p, m i R-484 \\
m i R-125 a-5 p\end{array}$ & 0.009585627 \\
\hline \multirow[t]{6}{*}{ DOWN_BP } & 0014854 & Response to inactivity & 4 & $m i R-133 b, m i R-206$ & 0.001989302 \\
\hline & 0014870 & Response to muscle inactivity & 4 & $\operatorname{miR}-1, \operatorname{miR}-133 b$ & 0.001989302 \\
\hline & 0014877 & $\begin{array}{l}\text { Response to muscle inactivity involved } \\
\text { in regulation of muscle adaptation }\end{array}$ & 4 & $m i R-206, m i R-1$ & 0.001989302 \\
\hline & 0014894 & $\begin{array}{l}\text { Response to denervation involved } \\
\text { in regulation of muscle adaptation }\end{array}$ & 4 & $\operatorname{miR}-1, \operatorname{miR}-133 b$ & 0.001989302 \\
\hline & 0002368 & B cell cytokine production & 2 & $m i R-206, m i R-1$ & 0.002474699 \\
\hline & 0002424 & $\begin{array}{l}\text { T cell mediated immune response } \\
\text { to tumor cell }\end{array}$ & 2 & $m i R-1, m i R-206$ & 0.002474699 \\
\hline \multirow[t]{6}{*}{ DOWN_MF } & 0005008 & $\begin{array}{l}\text { Hepatocyte growth factor-activated } \\
\text { receptor activity }\end{array}$ & 4 & $m i R-133 b, m i R-206$ & 0.001415624 \\
\hline & 0030955 & Potassium ion binding & 4 & $m i R-206, m i R-140-3 p$ & 0.006643278 \\
\hline & 0031420 & Alkali metal ion binding & 4 & $m i R-133 b, m i R-1$ & 0.007459611 \\
\hline & 0003688 & DNA replication origin binding & 2 & $m i R-206, m i R-1$ & 0.014287542 \\
\hline & 0031078 & $\begin{array}{l}\text { Histone deacetylase activity } \\
\text { (H3-K14 specific) }\end{array}$ & 4 & $m i R-206, m i R-140-3 p$ & 0.016613831 \\
\hline & 0032041 & $\begin{array}{l}\text { NAD-dependent histone deacetylase } \\
\text { activity (H3-K14 specific) }\end{array}$ & 4 & $m i R-1, m i R-206$ & 0.016613831 \\
\hline
\end{tabular}

DEG, differentially expressed gene; BP, biological process; MF, molecular function.

In the PPI network of the DEGs, MAD2L1 and AURKA were with high degrees. The overexpression of Mad2 can induce early dyscrasia, lung metastasis and poor survival in OS (29). The knockdown of Mad2 leads to OS cell death through apoptosis associated with Rad21 cleavage; thus, Mad2 may serve as a target for cancer therapy (30). AURKA can promote cell cycle and suppress cell apoptosis, and the inhibition of AURKA by specific short hairpin RNA (shRNA) may 
be a promising therapeutic strategy of OS (31). Furthermore, in the TF-DEG regulatory network, the TF, STAT3, targeted the most DEGs. By binding to the promoter region of $m i R-125 b$ and acting as a transactivator, STAT3 regulates $m i R-125 b$ which serves as a potential target in the therapy of OS (32). The overexpression of phosphorylated-STAT3 in OS cells is implicated in poor prognosis and may function as a prognostic indicator and therapeutic target for OS $(33,34)$. These data suggest that MAD2L1, AURKA and STAT3 may be closely associated with OS.

Some other molecules have also been involved in OS. The deregulation of $m i R-1$ and $m i R-133 b$ may correlate with cell cycle and cell proliferation of OS by mediating c-met (MET) protein expression (35). Through directly regulating PTEN/AKT signaling, miR-128 functions in the proliferation of human OS cells (36). The hypermethylation of p14ARF and ESR1 separately correlates with the absence of metastases at diagnoses and poor survival, therefore, p14ARF and ESR1 hypermethylation may be used as prognostic indicators for in OS (37). In 143B OS cells, phosphorylated and activated $c$-Jun and Fra- 1 (also known as FOSLI) can induce $M M P 1$ gene expression which may be a target for invasive and pulmonary metastases of OS, therefore, phosphorylated c-Jun and Fra-1 may affect invasion of OS through mediating $M M P 1$ (38).

In conclusion, this study identified key genes or miRNAs involved in OS. We screened 1,609 DEGs and 149 DE-miRNAs in the OS cell lines compared with normal bones. Besides, some molecules may correlate with OS, such as MAD2L1, AURKA, STAT3, ESR1, FOSL1, miR-1 and miR-128. However, experimental researches are still necessary to validate the functions of these molecules in OS.

\section{Competing interests}

The authors declare that they have no competing interests.

\section{References}

1. Wittig JC, Bickels J, Priebat D, Jelinek J, Kellar-Graney K, Shmookler B and Malawer MM: Osteosarcoma: A multidisciplinary approach to diagnosis and treatment. Am Fam Physician 65: 1123-1132, 2002.

2. Ottaviani $\mathrm{G}$ and Jaffe N: The epidemiology of osteosarcoma. In: Pediatric and Adolescent Osteosarcoma. Springer, pp3-13, 2010.

3. Stiller CA, Bielack SS, Jundt G and Steliarova-Foucher E: Bone tumours in European children and adolescents, 1978-1997. Report from the Automated Childhood Cancer Information System project. Eur J Cancer 42: 2124-2135, 2006.

4. Stiller CA, Craft AW and Corazziari I; EUROCARE Working Group: Survival of children with bone sarcoma in Europe since 1978: Results from the EUROCARE study. Eur J Cancer 37: 760-766, 2001.

5. Maire G, Yoshimoto M, Chilton-MacNeill S, Thorner PS Zielenska M and Squire JA: Recurrent RECQL4 imbalance and increased gene expression levels are associated with structural chromosomal instability in sporadic osteosarcoma. Neoplasia 11: 260-268, 2009

6. Zhou H, Randall RL, Brothman AR, Maxwell T, Coffin CM and Goldsby RE: Her-2/neu expression in osteosarcoma increases risk of lung metastasis and can be associated with gene amplification. J Pediatr Hematol Oncol 25: 27-32, 2003.

7. Sadikovic B, Thorner P, Chilton-Macneill S, Martin JW, Cervigne NK, Squire J and Zielenska M: Expression analysis of genes associated with human osteosarcoma tumors shows correlation of RUNX2 overexpression with poor response to chemotherapy. BMC Cancer 10: 202, 2010
8. Gamberi G, Benassi MS, Bohling T, Ragazzini P, Molendini L Sollazzo MR, Pompetti F, Merli M, Magagnoli G, Balladelli A, et al: C-myc and c-fos in human osteosarcoma: Prognostic value of mRNA and protein expression. Oncology 55: 556-563, 1998.

9. Lu XY, Lu Y, Zhao YJ, Jaeweon K, Kang J, Xiao-Nan L, Ge G, Meyer R, Perlaky L, Hicks J, et al: Cell cycle regulator gene CDC5L, a potential target for 6p12-p21 amplicon in osteosarcoma. Mol Cancer Res 6: 937-946, 2008.

10. de Nigris F, Botti C, de Chiara A, Rossiello R, Apice G, Fazioli F, Fiorito C, Sica V and Napoli C: Expression of transcription factor Yin Yang 1 in human osteosarcomas. Eur J Cancer 42: 2420-2424, 2006.

11. Cao Y, Zhou Z, de Crombrugghe B, Nakashima K, Guan H, Duan X, Jia SF and Kleinerman ES: Osterix, a transcription factor for osteoblast differentiation, mediates antitumor activity in murine osteosarcoma. Cancer Res 65: 1124-1128, 2005.

12. Osaki M, Takeshita F, Sugimoto Y, Kosaka N, Yamamoto Y, Yoshioka Y, Kobayashi E, Yamada T, Kawai A, Inoue T, et al: MicroRNA-143 regulates human osteosarcoma metastasis by regulating matrix metalloprotease-13 expression. Mol Ther 19: 1123-1130, 2011.

13. Zhang H, Cai X, Wang Y, Tang H, Tong D and Ji F: microRNA-143, downregulated in osteosarcoma, promotes apoptosis and suppresses tumorigenicity by targeting Bcl-2. Oncol Rep 24: $1363-1369,2010$

14. Duan Z, Choy E, Harmon D, Liu X, Susa M, Mankin H and Hornicek F: MicroRNA-199a-3p is downregulated in human osteosarcoma and regulates cell proliferation and migration. Mol Cancer Ther 10: 1337-1345, 2011.

15. Ziyan W, Shuhua Y, Xiufang W and Xiaoyun L: MicroRNA-21 is involved in osteosarcoma cell invasion and migration. Med Oncol 28: 1469-1474, 2011.

16. Yan K, Gao J, Yang T, Ma Q, Qiu X, Fan Q and Ma B: MicroRNA-34a inhibits the proliferation and metastasis of osteosarcoma cells both in vitro and in vivo. PLoS One 7: e33778, 2012.

17. Namløs HM, Meza-Zepeda LA, Barøy T, Østensen IH, Kresse SH, Kuijjer ML, Serra M, Bürger H, Cleton-Jansen AM and Myklebost O: Modulation of the osteosarcoma expression phenotype by microRNAs. PLoS One 7: e48086, 2012.

18. Smyth GK: Limma: linear models for microarray data. In: Bioinformatics and computational biology solutions using $\mathrm{R}$ and Bioconductor. Springer, pp397-420, 2005.

19. Zhao M, Sun J and Zhao Z: TSGene: A web resource for tumor suppressor genes. Nucleic Acids Res 41: D970-D976, 2013

20. Chen JS, Hung WS, Chan HH, Tsai SJ and Sun HS: In silico identification of oncogenic potential of fyn-related kinase in hepatocellular carcinoma. Bioinformatics 29: 420-427, 2013.

21. Boyle EI, Weng S, Gollub J, Jin H, Botstein D, Cherry JM and Sherlock G: GO:TermFinder - open source software for accessing Gene Ontology information and finding significantly enriched Gene Ontology terms associated with a list of genes. Bioinformatics 20: 3710-3715, 2004

22. Kanehisa M, Goto S, Furumichi M, Tanabe M and Hirakawa M: KEGG for representation and analysis of molecular networks involving diseases and drugs. Nucleic Acids Res 38: D355-D360, 2010.

23. Franceschini A, Szklarczyk D, Frankild S, Kuhn M, Simonovic M, Roth A, Lin J, Minguez P, Bork P, von Mering C, et al: STRING v9.1: Protein-protein interaction networks, with increased coverage and integration. Nucleic Acids Res 41: D808-D815, 2013

24. Kohl M, Wiese S and Warscheid B: Cytoscape: software for visualization and analysis of biological networks. In: Data Mining in Proteomics. Springer, pp291-303, 2011.

25. Nepusz T, Yu H and Paccanaro A: Detecting overlapping protein complexes in protein-protein interaction networks. Nat Methods 9: 471-472, 2012.

26. Fujita PA, Rhead B, Zweig AS, Hinrichs AS, Karolchik D, Cline MS, Goldman M, Barber GP, Clawson H, Coelho A, et al: The UCSC genome browser database: Update 2011. Nucleic Acids Res 39: D876-D882, 2011.

27. Xiao F, Zuo Z, Cai G, Kang S, Gao X and Li T: miRecords: An integrated resource for microRNA-target interactions. Nucleic Acids Res 37: D105-D110, 2009.

28. Dweep H, Sticht C, Pandey P and Gretz N: miRWalk - database: Prediction of possible miRNA binding sites by 'walking' the genes of three genomes. J Biomed Inform 44: 839-847, 2011. 
29. Yu L, Liu S, Guo W, Zhang B, Liang Y and Feng Q: Upregulation of Mad2 facilitates in vivo and in vitro osteosarcoma progression. Oncol Rep 28: 2170-2176, 2012.

30. Yu L, Guo W, Zhao S, Tang J and Liu J: Knockdown of Mad2 induces osteosarcoma cell apoptosis-involved $\operatorname{Rad} 21$ cleavage. J Orthop Sci 16: 814-820, 2011.

31. Jiang Z, Jiang J, Yang H, Ge Z, Wang Q, Zhang L, Wu C and Wang J: Silencing of Aurora kinase A by RNA interference inhibits tumor growth in human osteosarcoma cells by inducing apoptosis and G2/M cell cycle arrest. Oncol Rep 31: 1249-1254, 2014.

32. Liu LH, Li H, Li JP, Zhong H, Zhang HC, Chen J and Xiao T: miR-125b suppresses the proliferation and migration of osteosarcoma cells through downregulation of STAT3. Biochem Biophys Res Commun 416: 31-38, 2011.

33. Ryu K, Choy E, Yang C, Susa M, Hornicek FJ, Mankin H and Duan Z: Activation of signal transducer and activator of transcription 3 (Stat3) pathway in osteosarcoma cells and overexpression of phosphorylated-Stat 3 correlates with poor prognosis. J Orthop Res 28: 971-978, 2010.

34. Wang YC, Zheng LH, Ma BA, Zhou Y, Zhang MH, Zhang DZ and Fan QY: Clinical value of signal transducers and activators of transcription 3 (STAT3) gene expression in human osteosarcoma. Acta Histochem 113: 402-408, 2011.
35. Novello C, Pazzaglia L, Cingolani C, Conti A, Quattrini I, Manara MC, Tognon M,Picci P and Benassi MS: miRNA expression profile in human osteosarcoma: Role of miR-1 and miR-133b in proliferation and cell cycle control. Int J Oncol 42: 667-675, 2013.

36. Shen L, Chen XD and Zhang YH: MicroRNA-128 promotes proliferation in osteosarcoma cells by downregulating PTEN. Tumour Biol 35: 2069-2074, 2014.

37. Sonaglio V, de Carvalho AC, Toledo SR, Salinas-Souza C, Carvalho AL, Petrilli AS, de Camargo B and Vettore AL: Aberrant DNA methylation of ESR1 and p14ARF genes could be useful as prognostic indicators in osteosarcoma. Onco Targets Ther 6: 713-723, 2013.

38. Kimura R, Ishikawa C, Rokkaku T, Janknecht R and Mori N: Phosphorylated c-Jun and Fra-1 induce matrix metalloproteinase-1 and thereby regulate invasion activity of 143B osteosarcoma cells. Biochim Biophys Acta 1813: 1543-1553, 2011.

(i) $(9)$ This work is licensed under a Creative Commons Attribution-NonCommercial-NoDerivatives 4.0 International (CC BY-NC-ND 4.0) License. 